\section{Trabalho, saúde e vulnerabilidade na pandemia de COVID-19}

\author{
Labor, health and vulnerability in the COVID-19 \\ pandemic
}

Trabajo, salud y vulnerabilidad en la pandemia

de COVID-19 


\section{Introdução}

A COVID-19, doença causada pelo novo coronavírus (SARS-CoV-2), declarada como pandemia pela Organização Mundial da Saúde (OMS), tem configurado uma crise humanitária pela sua alta transmissibilidade e impactos sociais e econômicos. As pesquisas científicas têm avançado na caracterização do vírus, infecção e casos, nas vias de transmissão e medidas de prevenção, contenção e controle da doença. Entretanto, ainda existem muitas lacunas em relação ao perfil epidemiológico dos casos e óbitos, bem como do contexto de desigualdades sociais que perpassam o nível de exposição e a capacidade de proteção ao vírus e mitigação dos efeitos sociais provocados pela pandemia.

O trabalho assume centralidade na análise de estratégias de controle da doença e retomada de desenvolvimento econômico no período pós-pandemia, viabilizando, ou não, a manutenção do distanciamento social e de condições dignas de sobrevivência. Essas condições poderiam ser alcançadas por meio da garantia de renda e direitos sociais, e a proteção à saúde de trabalhadoras(es) envolvidas(os) em atividades essenciais, como atenção à saúde, produção e distribuição de alimentos, limpeza urbana, dentre outras. De acordo com o Decreto no 10.282 1, de 20 de março de 2020, do Governo Federal, no seu Art 3o $\$ 1$ 1o: "São serviços públicos e atividades essenciais aqueles indispensáveis ao atendimento das necessidades inadiáveis da comunidade...”. Algumas categorias de trabalhadores, apesar de não serem consideradas atividade essencial, permaneceram submetidas ao trabalho presencial sob o risco aumentado de exposição à COVID-19. Destacam-se entre essas, as trabalhadoras domésticas, categoria de risco ampliado para COVID-19 no contexto brasileiro 2,3.

Entre os primeiros casos registrados em dezembro de 2019 na cidade chinesa de Wuhan, aproximadamente metade destes (49\%) compartilhava alguma forma de exposição a um mercado de peixes (Huanan Seafood Wholesale Market), incluindo trabalhadores, consumidores e moradores do entorno do mercado 4. Desde então, até 30 de agosto de 2020, já são mais de 24 milhões de casos e mais de 838 mil óbitos em quase todos os países do mundo 5. A pandemia se apresenta em diferentes fases no mundo e, em algumas regiões, a flexibilização das medidas de contingenciamento passa a ser cogitada como forma de garantir a retomada econômica. A Organização Internacional do Trabalho (OIT) manifesta preocupação com esse momento de transição, e afirma que são necessários controles específicos e medidas amplas de prevenção e vigilância para a redução de risco de uma segunda onda de contágio no local de trabalho. A OIT reafirma o contexto de trabalho como foco estratégico de prevenção e controle da doença, pela natureza relacional do trabalho in loco, no contexto familiar ou no ambiente comunitário 6 .

O distanciamento social, estratégia principal de mitigação da pandemia, seguida pelos países afetados em diferentes níveis, apesar de obrigatório para a maioria das atividades econômicas e sociais, com exceção de atividades essenciais, se apresenta restrito a grupos em situação mais estável, como os servidores públicos ou trabalhadoras(es) formais, que possuem algum tipo de proteção previdenciária ou trabalhista. Assim, o trabalho assume papel relevante na efetividade do distanciamento, seja pela viabilidade de manutenção deste e das condições de vida permitidas pelo vínculo de trabalho, seja pela impossibilidade de adoção das medidas de proteção devido à precarização do trabalho, aos tipos de serviços a serem prestados e aos desafios para a sobrevivência do trabalhador 7 .

O eixo de argumentação deste ensaio, em torno do risco e vulnerabilidade de trabalhadores diante da crise sanitária e humanitária provocada pela pandemia de COVID-19, recupera a convergência construída na Saúde Coletiva em torno da proposição de Ayres et al. 8 sobre o modelo de vulnerabilidade, que engloba os planos de determinação da vulnerabilidade individual, social e programática. A discussão aqui desenvolvida privilegia os elementos da vulnerabilidade social, reconhecida como a que "está relacionada à possibilidade de o trabalhador perder o emprego ou sofrer forte redução de renda" 2 durante a pandemia e a vulnerabilidade programática, de insuficiência da resposta do Estado e empregadores às necessidades dessas populações, seja no âmbito das políticas sociais, seguridade social, assistência à saúde ou da proteção ao e no trabalho 9,10.

Desde junho de 2020, as estimativas já destacavam o Brasil como o epicentro na América do Sul e o segundo país em número de casos e óbitos em todo o mundo 11. A chegada da pandemia ao país agravou as desigualdades de uma conjuntura na qual trabalhadoras(es) acumulam perdas relevantes de direitos trabalhistas e previdenciários. Nesse sentido, a pandemia e suas repercussões sanitárias, econômicas e sociais aprofundaram um contexto de intensa fragilidade e desregulamentação 
do trabalho. Esse cenário tem como um dos marcos a reforma trabalhista, materializada na Lei no 13.467, de julho de 2017 12,13. Essa reforma legitimou as formas mais frágeis de contratualização do trabalho e os prejuízos ao emprego, com a criação de subcategorias de empregados mais vulneráveis em termos de direitos, destacando-se o trabalho intermitente e a terceirização de atividade-fim, tornando-a ilimitada 14.

Para o campo da Saúde do Trabalhador, a reforma trabalhista estabeleceu o cenário favorável à emergência de mais doenças e acidentes. É nessa conjuntura que chega a pandemia da COVID-19. Em meio à maior crise sanitária dos últimos cem anos, uma parcela de trabalhadoras(es) está em condições aviltantes de renda e moradia, entre outras adversidades do seu cotidiano, que implicam flagrante obstáculo às estratégias de contenção da pandemia e particularmente à opção do distanciamento social e da recomendação do "Fique em Casa". Nesse sentido, o objetivo deste ensaio foi discutir as repercussões da pandemia COVID-19 nas relações trabalho e saúde, sob a perspectiva dos riscos e vulnerabilidades de trabalhadores.

\section{Contextualização e construção das evidências sobre a relação trabalho e saúde na pandemia}

A construção do ensaio começou com base numa revisão da literatura sobre a interface saúde do trabalhador e COVID-19 nas bases PubMed, BIREME, Cochrane Library, medRxiv e LitCovid, bem como materiais na literatura cinza, incluindo trabalhos publicados entre dezembro de 2019 e abril de 2020. Utilizou-se como estratégia de busca: Workers OR Health Personnel OR Occupational Groups OR Community Health Workers AND COVID-19 OR severe acute respiratory syndrome coronavirus, adequando-as a cada base bibliográfica. Foram selecionadas 103 publicações científicas, além de relatórios de agências internacionais, como o Centro de Controle e Prevenção de Doenças dos Estados Unidos (CDC). A maioria das publicações $(82,3 \%)$ abordava profissionais da saúde, uso de equipamentos de proteção individual e exposição em ambiente hospitalar. O escopo de análise foi ampliado com perspectivas da sociologia do trabalho e de plataformas de vigilância epidemiológica e vigilância em saúde do trabalhador, ao exemplo da Plataforma Integrada de Vigilância em Saúde (Plataforma IVIS; http://plataforma.saude.gov.br/coronavirus/dados-abertos/) do Ministério da Saúde e do MonitoraCovid-19 (https://bigdata-covid19.icict.fiocruz.br/) da Fundação Oswaldo Cruz (Fiocruz), para melhor apreensão do objeto. Ademais, foi feita pesquisa documental com consulta a atos do poder público (leis, medidas provisórias e decisões judiciais) implementados no país e realizou-se a exploração de dados do Sistema de Vigilância Epidemiológica da Gripe (SIVEP-Gripe), disponíveis na Plataforma IVIS, utilizado pela vigilância epidemiológica para a notificação dos casos de síndrome respiratória aguda grave (SRAG), incluindo os casos de COVID-19, entre as etiologias da SRAG. A discussão do ensaio usou a perspectiva de vulnerabilidade no panorama da pandemia e o efeito nas relações de trabalho e saúde, bem como as possibilidades das respostas sociais apresentadas até o presente momento.

\section{Trabalho, saúde e vulnerabilidade: conceitos que se articulam}

A sociologia do trabalho aborda a vulnerabilidade como produto da precarização do trabalho, dada a centralidade do trabalho nos processos de sociabilidade. A perspectiva de agravamento da precarização do trabalho durante e após a pandemia aponta para a acentuação do cenário de desigualdades 15 anterior à mesma e surgimento de novos grupos sociais, historicamente mais desprotegidos, em situação de vulnerabilidade. Associado a isso, a inadequação das políticas sociais e dos dispositivos associados como respostas às demandas existentes pode constituir antipolíticas provenientes de "diagnósticos equivocados" que reduzem a capacidade distributiva e ampliam as desigualdades 16.

O conceito de vulnerabilidade proposto por Ayres et al. 8,17 reconhece as susceptibilidades populacionais e a resposta social, recorrendo à análise do risco, conceito clássico da epidemiologia, como indicador de problema ou medida (necessidade de saúde), e a capacidade de resposta social de diferentes grupos populacionais, da saúde pública e do Estado. Ao tratar dos riscos e vulnerabilidades 
de trabalhadores no contexto da pandemia, este ensaio dialoga com aberturas produzidas por essa perspectiva, analisando não apenas "as associações probabilísticas da distribuição populacional da infeç̧ão entre diferentes condições objetivas e mensuráveis", mas considerando "a variabilidade e a dinâmica das variáveis utilizadas nas análises de risco à luz de seus significados sociais concretos" 17 (p. 399). Assim, o texto se articula em torno do eixo da vulnerabilidade expressa na distribuição do adoecimento e morte por COVID-19, quanto às variações por gênero, idade, ocupação, escolaridade, mas também aos efeitos da precarização do trabalho e da desproteção social do trabalho, com a amplificação do trabalho informal e prejuízos ao trabalho artesanal, que expressam a sobreposição dinâmica de determinantes sociais como raça, gênero e classe social 17.

No âmbito da Ecologia Política e no Direito Ambiental, "as vulnerabilidades são entendidas como decorrentes de modelos de desenvolvimento..." que admitem e naturalizam "os processos geradores de vulnerabilidades sociais (...), ao mesmo tempo em que as instituições responsáveis pela sua regulação e controle não atuam de forma efetiva, pelo menos para certos grupos e territórios" 18 (p. 44). As vulnerabilidades, portanto, nessa perspectiva, são "resultantes de gradientes ou diferenciais de exposição dos grupos que vivem mais à periferia social e econômica do desenvolvimento e acabam por arcar com as principais cargas ambientais. Riscos em contextos vulneráveis são, portanto, uma questão de (in)justiça ambiental" 19 (p. 81). Atualizações da noção de vulnerabilidade na Saúde Coletiva tomam como diferencial um elemento que reposiciona o foco desse debate. A adoção de termos que evidenciam os processos de vulnerabilização, como "populações vulnerabilizadas, ao invés de em vulnerabilidade" busca contrapor à "naturalização do termo e do diálogo com a noção de determinação social do processo saúde-doença” 9 (p. 45).

Um estudo conduzido pela Rede de Pesquisa Solidária avaliou "o padrão de vulnerabilidade" durante a pandemia no Brasil, e identificou que os impactos da COVID-19 no mundo do trabalho obedecem às desigualdades estruturais da sociedade brasileira. Os negros com vínculos de trabalho mais frágeis compõem a maior parte da informalidade. Destaque para as mulheres, particularmente as negras, que também são muito vulneráveis por integrar setores econômicos historicamente desregulamentados - como a prestação de serviços domésticos, atividade que, em larga medida, foi reduzida, com demissão expressiva de trabalhadoras(es) 2 .

A relação gênero e pandemia também é outro aspecto que precisa ser destacado. Embora se admita que homens apresentem maior gravidade e mortalidade pela COVID-19 20, pouco se sabe sobre como a pandemia tem afetado diferentemente homens e mulheres 21. Prevê-se que o distanciamento social tenha efeito relevante para as mulheres, uma vez que estas são as mais engajadas no cuidado informal nas famílias, o que pode limitar a capacidade de trabalho remunerado, implicando diminuição de renda e autonomia destas 21 , aumento de sobrecarga física e mental e até aumento do risco de violências 22. Além disso, as mulheres representam $70 \%$ das trabalhadoras de saúde na linha de frente na atenção à pandemia, enfrentando múltiplos riscos à sua saúde, bem-estar e segurança 22 . A resposta efetiva à pandemia implica o reconhecimento das diferenças na vulnerabilidade de gênero relacionadas à exposição ao vírus, acesso à proteção e tratamento, adoecimento e morte, bem como políticas de proteção social e segurança.

A inserção de mulheres no mercado de trabalho é marcada por desigualdades sociais, desde a composição de vínculos de trabalho, remuneração e responsabilidade doméstica. Dados do boletim epidemiológico do Ministério da Saúde revelam que para cada homem desempregado havia cinco mulheres nesta condição, no Brasil, no período entre 2012 e 2019. Quando empregadas, as mulheres ocupavam predominantemente o setor saúde, educação, serviço social e serviços de manutenção e trabalho doméstico, atividades com menor remuneração e piores condições de segurança, algumas consideradas essenciais durante a pandemia e que implicam maior risco para a saúde das trabalhadoras 23. No contexto da COVID-19, a responsabilidade pelas atividades domésticas associada às multitarefas vinculadas ao cuidado, supervisão de filhos, estruturalmente determinada como papel feminino, além da provisão de sustento, expõe de forma desigual as mulheres não apenas ao risco da doença em si, mas também a repercussões na saúde física e mental.

Com base nos resultados da Pesquisa Nacional por Amostra de Domicílios Contínua (PNAD Contínua) do Instituto Brasileiro de Geografia e Estatística (IBGE), durante o distanciamento social no Brasil, 736 mil trabalhadores domésticos perderam seus empregos, entre os formais e os informais, predominando amplamente entre estes os vínculos mais precarizados 24 . As autoras pontuam que "considerando que 92,4\% dos empregados domésticos são mulheres e que provavelmente essa proporção se mantém entre os 
desempregados, podemos inferir que cerca de 680 mil trabalhadoras domésticas estão desempregadas no país" 24. Esse quadro aprofunda a situação de vulnerabilidade dessas trabalhadoras e suas famílias, considerando que grande parte delas é o principal provedor do núcleo familiar.

O relatório da OIT 6 estima que de um total de 292 milhões de pessoas empregadas na América Latina e no Caribe, 158 milhões trabalham em condições de informalidade, o equivalente a 54\%. O documento também afirma que as mulheres estão mais expostas à informalidade em regiões de baixa renda e geralmente são mais susceptíveis a situações vulneráveis do que seus parceiros. No Brasil, 38 milhões de trabalhadoras(es) se encontram no mercado informal, cerca de 41,4\% da população economicamente ativa 25 , cuja inserção instável e irregular pode colocá-las(os) sob maior risco de exposição.

Paralelo a esse cenário, ainda estão sob risco os trabalhadores que compõem os serviços essenciais. Esses serviços são aqueles considerados indispensáveis ao atendimento das necessidades da população ${ }^{1}$. Além das(os) trabalhadoras(es) da saúde, pode-se mencionar os serviços de proteção (policiais, agentes penitenciários, bombeiros), ocupações de escritório e apoio administrativo (bancários, correios e mensageiros, representantes de atendimento ao paciente), ocupações de serviços comunitários e sociais (assistentes sociais, conselheiros) e até ocupações de construção e extração (encanadores, instaladores de fossas sépticas, reparo de elevadores) 26. Essas ocupações devem ser contempladas pelas intervenções em saúde pública, já que podem constituir fontes potenciais de exposição ao vírus 3 .

Além do risco de contaminação, é preciso destacar os efeitos imediatos de ansiedade e de estresse entre trabalhadoras(es). Um estudo que avaliou profissionais de saúde identificou que 39\% apresentavam algum sofrimento psíquico, especialmente aqueles que trabalhavam em Wuhan com sobrecarga de trabalho 27. Esses profissionais também podem sentir medo do contágio e da transmissão para suas famílias, amigos ou colegas 28 , além de apresentarem sinais de esgotamento, como distúrbios do sono 29 . Apesar das ocupações ditas como linha de frente apresentarem alto risco para algum comprometimento na saúde mental, os impactos psicológicos das medidas de distanciamento social também trazem repercussões importantes para outras(os) trabalhadoras(es). Pessoas fisicamente ativas, mas que deixaram de trabalhar em decorrência da necessidade do distanciamento social, apresentaram piores condições de saúde física e mental ${ }^{30}$. Essas informações enfatizam a importância de apoiar as(os) trabalhadoras(es) por meio de intervenções de proteção à saúde mental em momentos de crise generalizada 28 .

Reconhecemos que a pandemia de COVID-19 vem atingindo trabalhadores de ocupações diversas, embora acometa predominantemente pessoas não brancas e migrantes, que estão em grupos socioeconômicos mais baixos, com acesso limitado a serviços de saúde ou que trabalham sem proteção social. Mesmo assim, as políticas instituídas não têm alcançado prioritariamente esses grupos mais vulneráveis 31. A OIT destaca a importância de ações voltadas para esses grupos, principalmente os que estão na economia informal, migrantes e domésticas. Sugere medidas de prevenção como capacitação e educação sobre práticas de trabalho seguras e saudáveis, concessão de equipamentos de proteção individual (EPIs) adequados, acesso a serviços de saúde e fornecimento de meios de subsistência alternativos. Adicionalmente, recomenda o mapeamento de atividades de risco e a capacitação de trabalhadores com atividades educativas a fim de garantir o trabalho seguro para o retorno ao trabalho no período de transição e pós-pandemia 32. É válido ressaltar que a maioria das medidas diz respeito ao trabalhador formal.

Uma pandemia naturalmente causa impactos na saúde, no emprego e na renda, no entanto, estes podem ser minimizados quando a conjuntura favorece a garantia de direitos sociais. No Brasil, as mudanças e o acúmulo de perdas intensificaram com a reforma trabalhista, que alterou capítulos da Consolidação das Leis do Trabalho e inaugurou um cenário no qual as mudanças alcançaram o paradigma constitucional de proteção do trabalho. O Direito do Trabalho, que se destacou e se distinguiu do Direito Civil, é baseado exatamente no reconhecimento da hipossuficiência do trabalhador, em sua condição vulnerável e subordinada, e é esta que é negada no bojo da reforma trabalhista no país 14. A precarização do trabalho e seu impacto no direito à saúde de trabalhadoras(es) já vinham sendo expressos no aumento do adoecimento físico e psíquico decorrente da fragilidade do controle social exercido para a adequação das condições laborais e prevenção de doenças e acidentes. Ademais, sem os dispositivos coletivos de organização de trabalhadoras(es), com sindicatos cada vez mais fragilizados, as condições favoráveis à sobrecarga de trabalho 12,14,33 e às vulnerabilidades ampliaram. 


\section{Panorama da pandemia no contexto de vulnerabilidade e trabalho}

A pandemia afetou de modo desigual diferentes grupos populacionais, porém pouco se produziu de evidências científicas sobre a saúde de trabalhadoras(es), em especial sobre aquelas(es) que laboram fora do Setor Saúde. Reforçando a existência dessa lacuna, foram encontradas 103 publicações sobre essa temática, sendo que a maioria reportava aspectos relacionados a trabalhadores da saúde e apenas $19(18,4 \%)$ discutiam sobre outros trabalhadores que não os da saúde. Esses achados evidenciam a invisibilidade permanente de trabalhadoras(es) essenciais como os da limpeza urbana, empregadas domésticas, entregadores, motoboys, caixas de supermercado, trabalhadoras(es) da indústria de combustíveis, da indústria química produtora de matéria-prima para sanitizantes e medicamentos, dentre outros.

Desses achados, a maioria apontava maior risco entre trabalhadoras(es) da saúde. A incidência da doença em Wuhan foi estimada em 41,5 casos por milhão de habitantes na população geral e 130,5 casos/milhão de habitantes. Ou seja, entre trabalhadoras(es) de saúde o risco triplica em relação à população geral 25. Outro estudo, que avaliou casos confirmados de COVID-19 de seis países asiáticos (Hong Kong, Japão, Cingapura, Taiwan, Tailândia e Vietnã), identificou que 14,9\% deles possivelmente estavam relacionados ao trabalho. Os cinco grupos de ocupação com maior frequência de casos foram trabalhadoras(es) da área da saúde (22\%), motoristas e trabalhadoras(es) de transporte (18\%), trabalhadoras(es) de serviços e vendedores (18\%), pessoal de limpeza, empregadas(os) domésticas(os) (9\%) e trabalhadoras(es) de segurança pública (7\%) 34.

Uma investigação com dados de 44.672 casos confirmados em Wuhan observou que 22\% destes aconteceram entre agricultores, 7,7\% entre industriários e 3,8\% entre trabalhadoras(es) da saúde. A mortalidade foi de 0,3 pessoa/mês entre agricultores, 1,2 entre industriários e 0,06 entre trabalhadoras(es) de saúde, com letalidade de 1,4\%, 0,7\% e 0,3\%, respectivamente 35. Segundo essas evidências, embora o risco de adoecer (incidência) seja superior entre trabalhadoras(es) de saúde, o risco de morrer, que provavelmente está relacionado a outros determinantes que não apenas à maior probabilidade de exposição ao vírus ${ }^{36}$, pode ser superior em outras ocupações, quando comparadas ao trabalho na saúde.

Os dados disponíveis no Brasil quanto à notificação de casos entre grupos de trabalhadoras(es) ainda são incipientes e apresentam subnotificação expressiva, o que gera invisibilidade das ocupações mais acometidas. Em parte, esses eventos podem ser justificados pela maneira como se apresentam as fichas de notificação. Desde o início da pandemia no Brasil, as instruções do Ministério da Saúde relacionadas à notificação dos casos suspeitos e confirmados da COVID-19 sofreram mudanças, ao exemplo da transferência do uso da plataforma RedCAP para a plataforma e-Sus Notifica ${ }^{37}$. A plataforma e-Sus Notifica instruía, até agosto de 2020, o registro da ocupação exclusivamente às profissões de saúde e segurança, permanecendo a grave lacuna sobre a variável ocupação, na notificação de COVID-19, com as repercussões já conhecidas 38. Vale ressaltar que entre profissionais de saúde não estão incluídas(os) trabalhadoras(es) da saúde em geral, que incorporam pessoal de higienização, recepção e outras ocupações centrais nas unidades de saúde. Para os casos hospitalizados, na ficha de registro individual de casos de SRAG o campo ocupação existe, no entanto, não é garantia de preenchimento 39 .

Ao explorar os dados do SIVEP-Gripe, entre o mês de janeiro e o dia 16 de junho de 2020, com um recorte para as notificações entre indivíduos de 18 aos 70 anos, observou-se 135.528 hospitalizações para a SRAG, das quais apenas 3,8\% (5.182) apresentavam registro de ocupação. Dos casos de SRAG notificados, 512 tiveram classificação final como SRAG não-COVID-19, 61.719 casos foram confirmados por critérios laboratoriais para COVID-19, além de 73.297 casos de SRAG não especificada ou com classificação final ignorada no sistema. Dentre os casos COVID-19, o registro da ocupação foi realizado em 2.602 fichas, que correspondem a 4,2\%. A tendência de subnotificação da variável ocupação foi mantida nos registros de óbitos, com apenas 3,4\% de registro da ocupação para os óbitos por SRAG (868/25.187) e 3,8\% (642/16.932) para os casos de COVID-19 (Plataforma IVIS; http://plataforma.saude.gov.br/coronavirus/dados-abertos/, acessado em 20/Jun/2020).

A subnotificação da variável ocupação limita a vigilância em saúde do trabalhador e inviabiliza a análise de risco entre as categorias profissionais, além da compreensão das vulnerabilidades. Essa condição de ausência de registro tem sido frequentemente debatida no campo da saúde. Entretanto, 
em condições de pandemia, em que a tomada de decisão deve considerar a proteção de trabalhadoras(es) para a manutenção de serviços essenciais, prevenindo novos casos e ao mesmo tempo a redução da progressão da doença, a subnotificação e sub-registro das ocupações podem comprometer estratégias de enfrentamento e demarcar iniquidades em saúde.

Apesar da fragilidade dos dados com registro de ocupação, a análise do SIVEP-Gripe permitiu uma descrição preliminar dos casos entre trabalhadoras(es) (Plataforma IVIS; http://plataforma. saude.gov.br/coronavirus/dados-abertos/, acessado em 20/Jun/2020). Das(os) 5.182 trabalhadoras(es) investigadas(os) para SRAG, 24,2\% (1.252) eram trabalhadoras(es) da saúde. Destaque para técnicas(os) e auxiliares de enfermagem com 458 casos, 36,6\% do total entre trabalhadoras(es) de saúde, seguidos por enfermeiras(os) com 20,4\% (255) e médicas(os) com 15,6\% (196). Ao considerar a confirmação diagnóstica por critérios laboratoriais, 25,6\% (666) de trabalhadoras(es) confirmadas(os) com COVID-19 eram da saúde. Dentre os casos de COVID-19 registrados em trabalhadoras(es) de saúde, as principais ocupações foram: técnicas(os) e auxiliares de enfermagem com 33,3\% (222/666); enfermeiras(os) com 19,4\% (129/666); e médicas(os) com 16,2\% (108/666).

Dentre os registros de SRAG e COVID-19 entre os demais grupos ocupacionais, foi possível identificar alguns grandes grupos que se destacaram. Para as notificações por SRAG, os grandes grupos ocupacionais com mais registros foram: trabalhadoras(es) dos serviços e comércio 21,5\% (1.112), com destaque para o pessoal do comércio e empregadas domésticas, seguidas de trabalhadoras(es) da indústria com 19,6\% (1.016), destaque para motoristas de caminhões de transporte de carga e pedreiros (indústria da construção civil). Em relação aos casos confirmados de COVID-19, o perfil é semelhante: trabalhadoras(es) dos serviços e comércio com 20,2\% (525), trabalhadoras(es) da indústria com 18,7\% (488). Técnicas(os) de nível médio e trabalhadoras(es) da agropecuária também aparecem nas notificações, na ordem de grandes grupos ocupacionais com maior frequência de registros da SRAG por COVID-19.

Já na distribuição dos óbitos por SRAG e casos de COVID-19 confirmados, segundo grandes grupos ocupacionais, o percentual dos registros entre trabalhadoras(es) dos serviços e comércio e da indústria, variando entre 24 e 26\%, supera o das(os) profissionais das ciências e das artes (18 a 20\%), que inclui os profissionais de saúde.

Em função do relevante sub-registro da variável ocupação, os dados aqui apresentados devem ser verificados com grande parcimônia pela absoluta insuficiência de dados. Ademais, registros hospitalares revelam apenas os casos de maior gravidade da doença e limitam a avaliação de risco da doença entre trabalhadoras(es). Além disso, a subnotificação restringiu também a construção de indicadores de gravidade da doença e impossibilitou estimativas razoáveis de frequência do evento entre categorias profissionais.

A limitação dos resultados epidemiológicos descritos neste tópico pode ser um importante indicador da dificuldade que antecede a pandemia, com relação ao registro de ocupações, pela falta de prioridade dada pelos gestores dos sistemas de informação em saúde (SIS) e, por extensão, pela comunidade dos serviços de saúde que preenche os instrumentos, alimentando estes sistemas. O trabalho, central na vida dos sujeitos, que determina socialmente as formas desiguais de adoecer e morrer, ainda não encontra o lugar de destaque compatível com esta relevância nos SIS, nos serviços de saúde e nas políticas públicas. Portanto, a lacuna da variável ocupação nos SIS ou a ausência de seu preenchimento quando o campo existe revelam apenas uma pequena parte da invisibilidade do trabalho e de trabalhadoras(es) na sociedade.

Concordando com a existência dessa lacuna, o editorial do principal veículo de publicação científica em Saúde do Trabalhador no Brasil, a Revista Brasileira de Saúde Ocupacional, assinalou que embora informações em saúde no país habitualmente subsidiem a adoção de "medidas de controle e prevenção, assim como para o planejamento e alocação dos recursos" 3 (p. 2) em saúde, estas comumente não possibilitam desagregar no nível de ocupação. Com isso, não é possível avaliar se onde e em que circunstâncias os indivíduos testados positivos ou diagnosticados com a doença estavam trabalhando, nem permitem identificar focos de disseminação relacionados com atividades laborais.

É importante ressaltar que conhecer a distribuição e frequência da COVID-19 no mundo do trabalho tem como finalidade precípua promover o diagnóstico situacional da doença, identificando as categorias profissionais mais atingidas, com vistas às políticas de proteção de trabalhadoras(es), fortalecendo as medidas de prevenção 40 . A existência de dados de ocupação favoreceria o diagnóstico 
da situação durante a pandemia e ao mesmo tempo viabilizaria o acompanhamento das repercussões da pandemia nas categorias mais atingidas pela COVID-19, permitindo relacionar estes dados com outros impactos econômicos e sociais da pandemia no mundo do trabalho.

\section{Análise crítica das políticas de enfrentamento e mitigação da pandemia no que se refere ao mundo do trabalho, trabalhadoras(es) formais e informais}

As desigualdades sociais perpassam dimensões fundamentais para análise da evolução e mitigação da COVID-19 no contexto brasileiro. Populações de baixa renda, distribuídas de forma heterogênea pelas regiões do país, apresentam maior exposição ao novo coronavírus devido aos aglomerados urbanos, restrição ao saneamento básico, dependência de transporte público e nível de acesso aos serviços de saúde ${ }^{41}$. Nesse cenário, a desigualdade social e a elevada pobreza e miséria existentes no Brasil constituem um sinal de alerta maior quando comparados com outros países. A China, por exemplo, apresenta peculiaridades sociopolíticas e culturais que a colocam em situação mais favorável, além de dispor de recursos financeiros superiores aos nossos 42 . As iniquidades existentes no país certamente serão intensificadas em decorrência da redução dos fluxos econômicos. A informalidade, que representa aproximadamente $41,4 \%$ do mercado de trabalho brasileiro 25 , durante este período de crise sanitária e econômica figurará como um dos principais problemas relacionados à perda de renda, com ausência de dispositivos de proteção social e garantia de direitos trabalhistas e de seguridade social.

As políticas do governo federal adotadas em meio à pandemia, que deveriam ampliar a proteção social de trabalhadoras(es), não têm sido concebidas nesta perspectiva, segundo estudiosos do trabalho e do direito do trabalho. Essas medidas de governo explicitam a escolha de proteção do mercado e dos negócios em detrimento da proteção de trabalhadoras(es). A Medida Provisória (MP) no 927, publicada em 22 de março de 2020, chegou a prever a suspensão de contratos de trabalho e de remuneração pelo período de quatro meses, que deixaria trabalhadoras(es) "à própria sorte, agravando o caos social e econômico e institucionalizando um autêntico crime humanitário" 43. A extensa reação da sociedade, em meio à pandemia, obrigou a revogação do artigo 18 dessa MP, pelo seu proponente, o presidente da República do Brasil. Outros prejuízos do texto da MP estão mantidos e em vigor, e o Governo Federal publicou em 1 de abril de 2020 a MP 936 que viria corrigir a omissão da MP 927 quanto ao papel do Estado na preservação de empregos e renda na crise do coronavírus.

A MP então, instituiu, para trabalhadoras(es) formais o denominado Programa Emergencial de Manutenção do Emprego e da Renda, que dispõe sobre medidas trabalhistas complementares para o enfrentamento do estado de calamidade pública. A medida autoriza os empregadores a suspenderem o contrato de trabalho temporariamente, a reduzirem salários e jornadas (por até 90 dias) ou suspenderem contratos de trabalho por até 60 dias. Destaca-se que caso haja suspensão do contrato ou redução da jornada de trabalho, com corte salarial, a proposta é que este seja complementado por meio de liberação do seguro desemprego, na mesma proporção da redução salarial. Entretanto, nem todas(os) as(os) trabalhadoras(es) formais farão jus a essa complementação, haja vista ser condição sine qua non preencher, previamente, os requisitos à concessão do referido seguro. Ainda nessa MP, confere-se ao empregado intermitente, subcategoria criada na reforma trabalhista já mencionada, um benefício emergencial mensal no valor de $\mathrm{R} \$ 600,00$ (seiscentos reais) pelo período de três meses, tendo sido prorrogado por mais dois meses. Uma nova prorrogação está em discussão, dado o avanço progressivo e persistente da pandemia no Brasil, entretanto, encontra resistência tanto em relação ao prazo quanto ao valor concedido 44 . Mesmo com a prorrogação, o benefício representa uma renda bem abaixo do salário mínimo para o trabalhador intermitente e foram muitos os relatos de dificuldades de acesso a este. Cerca de 106 milhões de pessoas tentaram o cadastro, sendo 59 milhões elegíveis para receberem o auxílio, porém, até a terceira semana de maio, quase um mês depois da divulgação do auxílio, muitas famílias ainda não tinham recebido o recurso o que, provavelmente, as obrigou a se exporem a condições inseguras de trabalho para garantir sua subsistência em meio à pandemia 45 .

Pesquisadores, cientistas políticos e profissionais de saúde, ao longo da pandemia, têm evidenciado a grave insuficiência das políticas do Governo Federal no enfrentamento da crise sanitária e das suas consequências sociais e econômicas. A exoneração do ministro da saúde ainda nos primeiros momentos da pandemia (em 16 de abril de 2020), com sucessores cujas passagens curtíssimas pelo 
ministério não marcaram ações de combate à pandemia, e a mais recente vacância neste cargo, com a presença de um quadro interino procedente das forças armadas, sem qualquer plano para a pasta da saúde em meio à tragédia de mais de 100 mil mortos pela COVID-19, são fatos que se acumulam explicitando a ausência do comando nacional. Estados e municípios adotaram políticas diversas, incluindo diferentes tempos e modalidades de decretação e permanência do distanciamento social, com variados graus de adesão decorrentes da ausência de apoio e de uma política articulada pelo Governo Federal para o país 46.

Para Filgueiras \& Druck 46, "além de combater e sabotar o isolamento social, com ações e pronunciamentos exemplares do próprio presidente, o governo (...) dificultou e tentou impedir a sua execução, ao não viabilizar, com a amplitude e presteza necessárias, o auxílio financeiro aos que perderam o emprego (ou ocupação, no caso dos autônomos e informais) e aos mais fragilizados sob todos os aspectos (renda, moradia e condições sanitárias)". Eles chamam atenção para a omissão do governo frente à grave situação que chegou a comprometer a subsistência de diversas categorias de trabalhadores, mencionando os "trabalhadores do mundo das artes e do setor de entregas dominado pelos aplicativos", e complementam assinalando a característica do governo federal marcada pela "negação da gravidade da pandemia, contrapondo falsamente o seu combate à necessidade da economia continuar funcionando, como se não houvesse possibilidade do governo atuar nas duas frentes".

Nesse cenário, é preciso considerar ainda que a força de trabalho em saúde ocupa lugar de destaque, tendo em vista sua centralidade, especialmente reconhecida, na manutenção da rede de assistência. Em meio à crise sanitária que esta pandemia representa, a visibilidade alcançada pelo Sistema Único de Saúde (SUS) e pelas(os) trabalhadoras(es) da saúde expressa neste momento sua importância estrutural não reconhecida e, ao contrário, fortemente negada pelas políticas de governo nos últimos anos. É possível distinguir pelo menos duas perspectivas para o destaque dado neste momento aos profissionais de saúde. Por um lado, esse destaque está vinculado à sua imprescindibilidade no enfrentamento da pandemia, ou seja, manter a força de trabalho, evitando perdas de quadros, pelo adoecimento ou morte. Por outro, essa imprescindibilidade confere visibilidade ao trabalho em saúde, desvelando em parte os desafios cotidianamente presentes no mundo do trabalho, em geral, e no trabalho em saúde, em particular, mesmo que referido a profissionais de saúde e não a trabalhadoras(es) em saúde.

Independentemente de uma ou outra dessas duas perspectivas, evidencia-se que trabalhadoras(es) de saúde ganharam destaque, sendo alvo de diversas notas técnicas 23,47,48. Nessas notas, trabalhadoras(es) dos serviços de saúde são reconhecidas(os) como grupo vulnerável prioritário na pandemia da COVID-19. Embora tenha ganhado notoriedade neste período, a MP 927 do Governo Federal trazia no seu artigo 29 que: "Os casos de contaminação pelo coronavírus (COVID-19) não serão considerados ocupacionais, exceto mediante comprovação do nexo causal”. Criou-se uma incerteza, especialmente entre os profissionais de saúde e suas representações em sindicatos e conselhos, que interpretaram que esse artigo 29 criaria dificuldades para o reconhecimento da COVID-19 como doença relacionada ao trabalho.

A fim de contribuir no sentido de reafirmar os direitos de trabalhadoras(es) da saúde com relação à COVID-19, a Frente Ampla em Defesa da Saúde dos Trabalhadores instituiu em 2 de abril de 2020 o Grupo Técnico que elaborou uma Nota Técnica, publicada em 7 de abril de 2020, que visou a esclarecer as(os) trabalhadoras(es), suas representações sindicais e Conselhos Profissionais acerca da relação entre COVID-19 e o trabalho em saúde e afirma: "A definição do diagnóstico clínico-ocupacional de uma doença ou agravo requer a investigação da relação entre a doença e o trabalho (nexo-causal entre doença e trabalho), que é definido a partir da evidência de exposição ocupacional. A natureza do trabalho em saúde, que neste momento implica o cuidado a pessoas com a doença COVID-19 ou a realização de tarefas nos ambientes de trabalho de unidades de assistência à saúde (recepção, higienização, laboratório e afins), resulta na exposição ocupacional de trabalhadoras(es) deste Setor (...) - cuja presença e consequente exposição ocupacional são determinadas pela ocupação exercida. Portanto, o estabelecimento da relação com o trabalho, ou o nexo-causal entre COVID-19 e trabalho, para todas(os) trabalhadoras(es) em efetiva atividade ocupacional nas tarefas de cuidado a pessoas ou nas demais tarefas dentro dos locais de trabalho nos quais o cuidado é prestado, fica definido" 48.

A Norma Técnica da Frente Ampla é o documento que orienta medidas gerais de caráter preventivo à COVID-19, bem como direitos trabalhistas e previdenciários de portadores de COVID-19 relacionada ao trabalho entre trabalhadoras(es) da saúde, e tem sido seguida por profícuo debate 
acerca da necessidade de reconhecimento da relação da COVID-19 com o trabalho. A ampliação desse direito para trabalhadoras(es) em atividades essenciais ou para todas(os) trabalhadoras(es) que estão impossibilitadas(os) de adotarem o distanciamento social na pandemia em função das exigências de trabalho é que baseia esse debate.

Embora os potenciais riscos para as trabalhadoras da saúde estejam bem estabelecidos, cinco meses após o primeiro caso confirmado no Brasil, o então presidente ainda desrespeita este coletivo ao vetar integralmente o projeto de lei que previa pagamento de indenização aos trabalhadores incapacitados e aos familiares em caso de morte por COVID-19 decorrente do exercício profissional 49. Nesse sentido, ainda há muito a avançar quanto à temática, de modo a fortalecer os direitos das(os) trabalhadoras(es) e entender o papel central que o trabalho exerce no desenvolvimento humano, econômico e social de qualquer país.

\section{Considerações finais}

O conjunto de evidências reunidas neste texto acerca da COVID-19 no mundo do trabalho permite identificar as principais lacunas, desafios e possibilidades para a atuação dos serviços de saúde, da sociedade civil organizada e das universidades no difícil contexto da pandemia. As informações reunidas neste texto podem contribuir para a construção da agenda sobre pandemia e trabalho. Nesse sentido, a preservação da saúde de trabalhadoras(es) deve ser o eixo articulador das políticas públicas e das demais iniciativas. Assinalamos que trabalhadoras(es) que se mantêm em atividade de trabalho durante a pandemia - sem garantia do direito à saúde, pois este direito inclui a possibilidade de adoção do distanciamento social, que não se viabiliza em circunstâncias de iniquidades sociais e de saúde - devem ser prioritariamente alvo de atenção para controlar a disseminação da doença e proteger a vida.

Ampliar as políticas e medidas de proteção é uma necessidade urgente para outras atividades com risco de exposição, como é o caso de trabalhadoras(es) de farmácia, entregadores (delivery), carteiros, de transporte de cargas e de passageiros e pessoal de apoio, frentistas de postos de combustíveis, serviços de abastecimento e vendas de alimentos e de produtos; serviços residenciais, porteiros e zeladores, pessoal de limpeza, empregados(as) domésticos(as); vigilantes, policiais, bombeiros; cuidadores de idosos e de pessoas dependentes; trabalhadoras(es) de manutenção de serviços públicos e privados de telefonia, eletricidade, água, gás, Internet, segurança pública, serviços funerários, coleta de lixo, dentre outras.

É fundamental considerar ainda estratégias seguras para as(os) trabalhadoras(es) quando do retorno gradual às atividades, para além daquelas consideradas essenciais. É crucial que as medidas de prevenção da COVID-19 em locais de trabalho sejam adotadas, assegurando mudanças na gestão do trabalho e da produção, com redução de turnos e jornadas de trabalho, a fim de evitar aglomerações nesses locais e viabilizar a distância física mínima de dois metros para trabalhadoras(es) em atividade presencial. Disponibilidade de equipamentos de proteção coletiva e individual, com ênfase na proteção respiratória e fácil acesso às estações de higienização de mãos, é parte das medidas necessárias para a adequação dos locais de trabalho 50 . Ampliar o número de testes e manter os sistemas de vigilância continuada são objetivos da prevenção e controle da disseminação do vírus nos locais de trabalho.

Algumas experiências exitosas, particularmente nos serviços de saúde, em outras epidemias como a SARS, têm sido recomendadas como estratégias de enfrentamento e retomada de atividades. Destacamos a reorganização de fluxo de entrada e saída de usuários, testagem regular e em ambiente domiciliar de trabalhadoras(es), reorganização dos ambientes de trabalho, isolamento de setores específicos, orientação e estímulo ao uso de EPIs, redução da carga horária de trabalho, oferta de alojamento para a garantia de distanciamento social, oferta de apoio social e psicológico aos trabalhadores 51 .

A atual condição de crise humanitária provocada pela pandemia acelerou uma série de iniciativas de proteção social que teve centralidade no campo de trabalho. Vários governos implementaram ou estenderam programas de proteção a grupos vulneráveis, com introdução de medidas e legislação sobre assistência à saúde, mitigação do desemprego e assistência social, que incluíram desde a transferência de renda até o apoio e manutenção de postos de trabalho ${ }^{32}$. Prospectivamente, essas medidas 
devem ser incorporadas como mecanismos sustentáveis de proteção social, com participação de trabalhadoras(es), suas representações, empregadores e Estado, para a garantia da legislação trabalhista e previdenciária, com a formulação de uma agenda sólida de consolidação do trabalho decente e retomada do desenvolvimento pós-pandemia.

\section{Colaboradores}

K. O. B. Santos contribuiu com o planejamento e elaboração do estudo, escrita e revisão do manuscrito. R. C. P. Fernandes, M. M. C. Almeida e S. S. Miranda contribuíram com a escrita, revisão e discussão do artigo. Y. F. Mise contribuiu com a escrita e revisão do artigo. M. A. G. Lima contribuiu com o planejamento do estudo, escrita, revisão e discussão do artigo.

\section{Informações adicionais}

ORCID: Kionna Oliveira Bernardes Santos (00000003-3181-2696); Rita de Cássia Pereira Fernandes (0000-0002-3353-5365); Milena Maria Cordeiro de Almeida (0000-0001-8065-4298); Samilly Silva Miranda (0000-0002-1488-1246); Yukari Figueroa Mise (0000-0002-5273-1548); Monica Angelim Gomes de Lima (0000-0003-3364-8439).

\section{Agradecimentos}

Agradecemos a Erika dos Santos Aragão, coordenadora do grupo de Impactos Sociais e Distributivos RedeCOVida.

\section{Referências}

1. Brasil. Decreto no 10.282 , de 20 de março de 2020. Regulamenta a Lei no 13.979, de 6 de fevereiro de 2020, para definir os serviços públicos e as atividades essenciais. Diário Oficial da União 2020; 20 mar.

2. Arantes JT. Estudo avalia a vulnerabilidade de trabalhadores na crise causada pela pandemia de COVID-19. Agência FAPESP 2020; 30 abr. https://agencia.fapesp.br/estudo-avalia-a-vul nerabilidade-de-trabalhadores-na-crise-cau sada-pela-pandemia-de-covid-19/33065/.

3. Jackson Fiho JM, Assunção AÁ, Algranti E, Garcia EG, Saito CA, Maeno M. A saúde do trabalhador e o enfrentamento da COVID-19. Rev Bras Saúde Ocup 2020; 45:e14.

4. Yan Y, Shin WI, Pang YX, Meng Y, Lai J, You $\mathrm{C}$, et al. The first 75 days of novel coronavirus (SARS-CoV-2) outbreak: recent advances, prevention, and treatment. Int J Environ Res Public Health 2020; 17:2323.

5. World Health Organization. Coronavirus disease (COVID-19). Weekly epidemiological update. https://www.who.int/docs/de fault-source/coronaviruse/situation-reports/ 20200831-weekly-epi-update-3.pdf?sfvrsn= d7032a2a_4 (acessado em 20/Jun/2020).

6. International Labour Organization. COVID-19 crisis and the informal economy. Immediate responses and policy challenges. https://www.ilo.org/global/topics/employ ment-promotion/informal-economy/publi cations/WCMS_743623/lang--en/index.htm (acessado em 14/Jun/2020).

7. Rede Covida. Saúde do trabalhador: riscos e vulnerabilidades. Boletim CoVida 2020; (6). https://covid19br. org/main-site-covida/wp-content/ uploads/2020/06/Boletim-CoVida-64.pdf. 
8. Ayres JRCM, França Júnior I, Calazans GJ, Saletti Filho HC. O conceito de vulnerabilidade e as práticas de saúde: novas perspectivas e desafios. In: Czeresnia D, Freitas CM, organizadores. Promoção da saúde: conceitos, reflexões, tendências. Rio de Janeiro: Editora Fiocruz; 2003. p. 117-39.

9. Oliveira RG. Health practices in contexts of vulnerability and neglect of diseases, people and territories: potentialities and contradictions in health care for homeless people. Saúde Soc 2018; 27:37-50.

10. Muñoz Sánchez AI, Bertolozzi MR. Pode o conceito de vulnerabilidade apoiar a construção do conhecimento em Saúde Coletiva? Ciênc Saúde Colet 2007; 12:319-24.

11. World Health Organization. Coronavirus disease 2019 (COVID-19). Situation report - 98. https://www.who.int/docs/default-source/ coronaviruse/situation-reports/20200427sitrep-98-covid-19.pdf? sfvrsn $=90323472 \_4$ (acessado em 14/Jun/2020).

12. Krein JD, Oliveira RV, Filgueiras VA. As reformas trabalhistas: promessas e impactos na vida de quem trabalha. Caderno CRH 2019; 32:225-9.

13. Filgueiras VA, Lima UM, Souza IF. Os impactos jurídicos, econômicos e sociais das reformas trabalhistas. Caderno CRH 2019; 32:23152.

14. Druck G, Dutra R, Silva SC. The labor counter-reform: outsourcing and precarization as a rule. Caderno CRH 2019; 32:289-305.

15. Pires LN, Carvalho L, Xavier LL. COVID-19 e desigualdade no Brasil. Cebes 2020; 6 abr. http://cebes.org.br/2020/04/covid-19-e-desi gualdade-no-brasil/.

16. Ivo ABL. A reconversão do social: dilemas da redistribuição no tratamento focalizado. São Paulo Perspect 2004; 18:57-67.

17. Ayres JRCM, Calazans GJ, Saletti Filho HC, França-Júnior I. Risco, vulnerabilidade e práticas de prevenção e promoção da saúde. In: Campos GWS, Minayo MCS, Akerman M, Drumond Júnior M, Carvalho YM, organizadores. Tratado de Saúde Coletiva. São Paulo: Hucitec Editora/Rio de Janeiro: Editora Fiocruz; 2009. p. 375-417.

18. Porto MFS. Uma ecologia política dos riscos: princípios para integrarmos o local e o global na promoção da saúde e da justiça ambiental. 2a Ed. Rio de Janeiro: Editora Fiocruz; 2012.

19. Porto MFS. Riscos, incertezas e vulnerabilidades: transgênicos e os desafios para a ciência e a governança. Política \& Sociedade 2005; 4:77103.

20. Sharma G, Volgman AS, Michos ED. Sex differences in mortality from COVID-19 pandemic: are men vulnerable and women protected? JACC Case Rep 2020; 2:1407-10.

21. Wenham C, Smith J, Morgan R. COVID-19: the gendered impacts of the outbreak. Lancet 2020; 395:846-8.

22. UN Women. COVID-19 and ending violence against women and girls. New York: UN Women; 2020.
23. Agência Nacional de Vigilância Sanitária. Medidas de prevenção e controle que devem ser adotadas durante a assistência aos casos suspeitos ou confirmados de infecção pelo novo Coronavírus (SARS-CoV-2). Nota Técnica no 04/2020 GVIMS/GGTES/ ANVISA. https://www20.anvisa.gov.br/ segurancadopaciente/index.php/alertas? t a $\mathrm{k}=\mathrm{callelement} \&$ for mat $=$ raw \&item $\mathrm{id}=836 \&$ element $=$ f f85c494b-2b32-4109b8c1-083cca2b7db6\&method=download\&ar gs $[0]=5 \mathrm{da} 686556 \mathrm{~b} 937 \mathrm{e} 9 \mathrm{db0cea} 5439 \mathrm{~b} 8633 \mathrm{fd}$ (acessado em 14/Jun/2020).

24. Myrrha LJD, Queiroz SN, Silva PS. Impactos da Covid-19 no (des)emprego doméstico. O que já podemos ver? Demografia UFRN 2020; 8 jun. https://demografiaufrn. net/2020/06/08/covid19-des-emprego-do mestico/.

25. Instituto Brasileiro de Geografia e Estatística. Pesquisa Nacional por Amostra de Domicílios Contínua. Rio de Janeiro: Instituto Brasileiro de Geografia e Estatística; 2020.

26. Baker MG, Peckham TK, Seixas NS. Estimating the burden of United States workers exposed to infection or disease: a key factor in containing risk of COVID-19 infection. medRxiv 2020; 6 mar. https://www.medrxiv. org/content/10.1101/2020.03.02.20030288v1.

27. Dai Y, Hu G, Xiong H, Qiu H, Yuan X. Psychological impact of the coronavirus disease 2019 (COVID-19) outbreak on healthcare workers in China. medRxiv 2020; 6 mar. https://www. medrxiv.org/content/10.1101/2020.03.03.200 $30874 \mathrm{v} 1$.

28. Xiang YT, Yang Y, Li W, Zhang L, Zhang Q, Cheung $\mathrm{T}$, et al. Timely mental health care for the 2019 novel coronavirus outbreak is urgently needed. Lancet Psychiatry 2020; 7:2289.

29. Qi J, Xu J, Li B, Huang J, Yang Y, Zhang Z, et al. The evaluation of sleep disturbances for Chinese frontline medical workers under the outbreak of COVID-19. medRxiv 2020; 17 mar. https://www.medrxiv.org/content/10.1101/2 020.03.06.20031278v2.

30. Zhang SX, Wang Y, Rauch A, Wei F. Unprecedented disruption of lives and work: health, distress and life satisfaction of working adults in China one month into the COVID-19 outbreak. Psychiatry Res 2020; 288:112958.

31. Devakumar D, Shannon G, Bhopal SS, Abubakar I. Racism and discrimination in COVID-19 responses. Lancet 2020; 395:1194.

32. Organização Internacional do Trabalho. OIT: é necessário garantir a proteção dos trabalhadores agora e após o fim do confinamento. OIT Notícias 2020; 28 abr. https://www.ilo. org/brasilia/noticias/WCMS_743059/lang-pt/index.htm.

33. Galvão A, Castro B, Krein JD, Teixeira MO. Labor reform: precarious work and the challenges for unions. Caderno CRH 2019; 32:253-70. 
34. Lan F-Y, Wei C-F, Hsu Y-T, Christiani DC, Kales SN. Work-related Covid-19 transmission. medRxiv 2020; 20 abr. https://www.medrxiv.org/content/10.1101/2020.04.08.20058 297v2.

35. The Novel Coronavirus Pneumonia Emergency Response Epidemiology Team. Vital surveillances: the epidemiological characteristics of an outbreak of 2019 novel coronavirus diseases (COVID-19) - China, 2020. China CDC Wkly 2020; 2:113-22.

36. Centers of Disease Control and Prevention. COVID-19 in racial and ethnic minority groups. https://www.cdc.gov/ coronavirus/2019-ncov/need-extra-precau tions/racial-ethnic-minorities.html (acessado em 14/Jun/2020).

37. Ministério da Saúde. Ficha de notificação completa para casos suspeitos e prováveis de Novo Coronavírus (COVID-19). https://red cap.saude.gov.br/surveys/?s=3PRKP3CAJ3 (acessado em 14/Jun/2020).

38. Diretoria de Vigilância e Atenção à Saúde do Trabalhador, Secretaria da Saúde do Estado da Bahia. Orientações técnicas para a investigação e notificação de casos de Covid-19 relacionados ao trabalho. http://renastonline.ensp. fiocruz.br/sites/default/files/arquivos/recur sos/orientacoestecnicascovid-st_divast_final_ 2junho2020_1.pdf (acessado em 14/Jun/2020).

39. Ministério da Saúde. Ficha de registro individual - casos de síndrome respiratória aguda grave hospitalizado. http://189.28.128.100/ sivep-gripe/Ficha_SIVEP_GRIPE_SRAG_ Hospital_31_03_2020.pdf (acessado em 14/ Jun/2020).

40. Burdorf A, Porru F, Rugulies R. The COVID-19 (Coronavirus) pandemic: consequences for occupational health. Scand J Work Environ Health 2020; 46:229-30.

41. Bucchianeri GW. Is SARS a poor man's disease? Socioeconomic status and risk factors for SARS transmission. Forum Health Econ Policy 2010; 13(2). https://doi.org/10.2202/15589544.1209.

42. Dias BC. Pandemia da Covid-19 e um Brasil de desigualdades: populações vulneráveis e o risco de um genocídio relacionado à idade. https://www.abrasco.org.br/site/gtenvelhe cimentoesaudecoletiva/2020/03/31/pande mia-do-covid-19-e-um-brasil-de-desigualda des-populacoes-vulneraveis-e-o-risco-de-umgenocidio-relacionado-a-idade/ (acessado em 14/Jun/2020).

43. Maior JLS. MP 936: do pandemônio à razão? https://www.jorgesoutomaior.com/blog/mp936-do-pandemonio-a-razao (acessado em 14/Jun/2020).

44. Ideia legislativa que pede prorrogação do auxílio emergencial vai para CDH. Agência Senado 2020; 10 ago. https://www12.senado.leg.br/ noticias/materias/2020/08/10/ideia-legisla tiva-que-pede-prorrogacao-do-auxilio-emer gencial-vai-para-cdh.
45. Auxílio Emergencial: 9,7 milhões aguardam análise, diz Caixa. G1 2020; 24 mai. https:// g1.globo.com/economia/noticia/2020/05/24/ auxilio-emergencial-97-milhoes-aguardam-a nalise.ghtml.

46. Filgueiras L, Druck G. A mudança de conjuntura e a resiliência de Bolsonaro. Le Monde Diplomatique Brasil 2020; 15 jul. https://di plomatique.org.br/a-mudanca-de-conjuntura -e-a-resiliencia-de-bolsonaro/.

47. Secretaria da Saúde do Estado da Bahia. Nota Técnica COE-SAÚDE no 35 de 28 de março de 2020. Procedimentos para trabalhadores de saúde da SESAB com suspeita de COVID-19 e contactantes da área de saúde. http://www.saude.ba.gov.br/wp-content/ uploads/2020/04/NT-no-35-28.03-afasta mento-profissionais-de-saude-e-contactantes. pdf (acessado em 14/Jun/2020).

48. Frente Ampla em Defesa da Saúde dos Trabalhadores. Nota Técnica Conjunta (1): orientação sobre direitos de trabalhadoras e trabalhadores dos serviços de saúde, enquanto grupo vulnerável prioritário na pandemia da COVID-19. http://renastonline.ensp.fiocruz. $\mathrm{br} / \mathrm{sites} / \mathrm{default} /$ files/arquivos/noticias/no ta_tecnica_da_frente_ampla_direitos_tra balhadores_07_04_20.pdf (acessado em 14/ Jun/2020).

49. Júnior J, Librelon R. Bolsonaro veta indenização de R\$ 50 mil a profissionais de saúde vitimados pela Covid-19. Agência Câmara de Notícias 2020; 4 ago. https://www.camara.leg. $\mathrm{br} /$ noticias/681548-bolsonaro-veta-indeniza cao-de-r-50-mil-a-profissionais-de-saude-vi timados-pela-covid-19/.

50. U.S. Department of Labor. Guidance on preparing workplaces for COVID-19. https:// www.osha.gov/Publications/OSHA3990.pdf (acessado em 14/Jun/2020).

51. Heliotério MC, Lopes FQRS, Sousa CC, Souza FO, Freitas PSP, Sousa FNF, et al. COVID-19: por que a proteção da saúde dos trabalhadores e trabalhadoras da saúde é prioritária no combate à pandemia? SciELO Preprints 2020; 2 fev. https://preprints.scielo.org/index.php/ scielo/preprint/view/664/855. 


\section{Abstract}

This essay discusses the repercussions of the COVID-19 pandemic on the relation between labor and health, from the perspective of the workers' risk and vulnerability. The pandemic has represented a humanitarian crisis, since both the disease and the measures to contain it lead to persistent socioeconomic effects. In this context, the labor category has an important role, either because of the feasibility of maintaining the social distancing and living conditions allowed by the employment relationship, or because of the impossibility of adopting protection strategies due to job insecurity. The essay was initially built on the basis of a literature review on the interface between COVID-19 and workers' health, carried out from December 2019 to April 2020, on the PubMed, BIREME, Cochrane Library, medRxiv and LitCovid bases, as well as using gray literature. Health professionals are more affected, but they also have greater access to diagnosis; however, data are still scarce on other professional categories, as well as on the social determinants that lead to greater labor-related vulnerability. In Brazil, the pandemic coincides with a situation in which workers accumulate significant losses of labor and social security rights, in addition to pre-existing social inequalities, such as precarious housing, with greater exposure and risk. Although the pandemic is still evolving, social inequalities are expected to intensify, with the deep retraction of the economy, and workers must be a priority target of attention in the control and spread of the disease, in addition to being the axis for planning public social and health protection policies.

Working Conditions; Occupational Health; Pandemics; Vulnerability Analysis;

COVID-19

\section{Resumen}

Este ensayo discute las repercusiones de la pandemia COVID-19 en la relación trabajo y salud, bajo la perspectiva del riesgo y vulnerabilidad de los trabajadores. La pandemia se ha configurado como una crisis humanitaria, ya que tanto la enfermedad, como las medidas de contención de la misma, generan efectos socioeconómicos persistentes. En este contexto, la categoría trabajo asume un papel relevante, sea por la viabilidad del mantenimiento del distanciamiento social, así como por las condiciones de vida permitidas por el vínculo laboral, sea por la imposibilidad de adopción de estrategias de protección, debido a la precarización del trabajo. La construcción del ensayo se inició a partir de una revisión de la literatura con la interfaz COVID-19 y salud de los trabajadores, realizada de diciembre de 2019 a abril de 2020, en las bases PubMed, BIREME, Cochrane Library, medRxiv y LitCovid, así como en la literatura gris. Los profesionales de salud son los más afectados, pero también con mayor acceso al diagnóstico, persistiendo lagunas sobre las demás categorías profesionales, así como acerca de los determinantes sociales que implican una mayor vulnerabilidad, relacionada con el trabajo. La pandemia coincide en Brasil con una coyuntura en la que las trabajadoras(es) acumulan pérdidas relevantes de derechos laborales y seguridad social, sumados a las desigualdades sociales preexistentes, por ejemplo, de precariedad de vivienda o con mayor exposición y riesgo. A pesar de que la evolución de la pandemia todavía esté en curso, se prevé que las desigualdades sociales se intensificarán, por la profunda retracción de la economía. Por ello, los trabajadores deben ser objetivo prioritario de la atención en el control y diseminación de la enfermedad, además de ser eje articulador de las politicas públicas de protección social y salud.

Condiciones de Trabajo; Salud Laboral;

Pandemias; Análisis de Vulnerabilidad;

COVID-19
Recebido em 22/Jun/2020

Versão final reapresentada em 08/Set/2020

Aprovado em 08/Set/2020 